\title{
Active Sound Design Development Based on the Harmonics of Main Order From Engine Sound
}

\author{
SOYOUN MOON ${ }^{1}$, AES Member, SUNGHWAN PARK'ㄹ, DONGGUN PARK ${ }^{1}$, MYUNGHWAN YUN ${ }^{1} \dagger$, \\ (auditoryuserinterface@gmail.com)（pshtmr@snu.ac.kr) (donggun.park@snu.ac.kr) (mhy@snu.ac.kr) \\ KYONGJIN CHANG ${ }^{3}$, AND DONGCHUL PARK ${ }^{3}$ \\ (changkj@hyundai.com) (dc.park@hyundai.com) \\ ${ }^{1}$ Department of Industrial Engineering \& Institute for Industrial System Innovation, Seoul National University, Seoul, \\ Republic of Korea \\ ${ }^{2}$ Interdisciplinary Program in Cognitive Science, Seoul National University, Seoul, Republic of Korea \\ ${ }^{3}$ Sound Design Research Lab, Hyundai Motors R\&D Center, Hwaseong, Republic of Korea
}

\begin{abstract}
Most previous studies on active sound design (ASD) development proposed regression models based on psychoacoustic parameters for engine sound design. However, order-based parameters are required for a real ASD development, considering that an ASD system is controlled by order levels. In this paper, we propose a regression model utilizing order-levelbased parameters that can be efficiently applied to ASD development. A jury test was conducted for 27 engine sound recordings using 36 participants with normal hearing ability to evaluate the level of affective adjectives. Then, acoustic parameters were measured from the engine sound recordings to identify the relationship between the adjectives and parameters. Finally, a regression model was derived through statistical analysis. The properties of the model were compared with those of models proposed in previous studies to verify its superiority. The proposed regression model can reduce the time and effort required for ASD development.
\end{abstract}

\section{O INTRODUCTION}

Recently, car makers have focused on representing their brand identities through active sound design (ASD) systems, which can be applied to an infotainment system without changing engine-related hardware [28]. To represent brand identity using an ASD system, understanding the relationship between affect and the acoustic parameters of engine sound is critical.

Some previous studies proposed regression models that are composed of psychoacoustic parameters. These models could be useful to elucidate the relationship between affect and psychoacoustic parameters for engine sounds. However, an order-level-based regression model is needed for actual ASD development considering that an ASD system is controlled by a tuning tool that adjusts engine order levels.

${ }^{\dagger}$ Corresponding author

*Co-corresponding author
To construct a regression model for engine sound, meaningful parameters of minimum numbers must be extracted without losing information about the sound character of the engine sound. In this paper, the harmonics pattern of an instrument is proposed as a reference structure to extract meaningful orders from engine sound. The concept was referred and applied to engine sound analysis to derive orders that can be used as parameters for the regression model.

In this context, we propose regression models based on the harmonics of main orders and overall level (SPL), which can be applied to the actual development of ASD systems. To achieve this, 27 engine sounds were recorded and tested by 36 participants to measure the subjective levels of two affective adjectives-Powerful and Refined.

Subsequently, two regression equations were derived from a statistical analysis of the two affective adjectives, which can be used for engine sound evaluation and design.

The proposed regression model represents brand identity with an ASD system by adjusting the order levels, yielding the expected levels of Powerful and Refined. Using these 
equations, the development of a more efficient ASD system that reduces the required time and effort can be achieved.

\section{REGRESSION MODEL FOR ENGINE SOUND DESIGN}

\subsection{Regression Model}

Regression analysis is a statistic method to find the functional relationship among variables. The relationship is expressed in the form of an equation or a model connecting the dependent (Response) variables and independent (Predict) variables. For a dependent variable $Y$ and the set of independent variables $X_{1}, X_{2}, \ldots, X_{p}$ ( $p$ denotes the number of independent variables), the relationship between $Y$ and $X_{1}, X_{2}, \ldots, X_{p}$ can be approximated by the regression model given below.

$$
Y=\beta_{0}+\beta_{1} X_{1}+\beta_{2} X_{2}+\cdots \beta_{p} X_{p}+\varepsilon
$$

where $\varepsilon$ is a random error representing the discrepancy in the approximation and $\beta_{0}, \beta_{1}, \beta_{2}, \ldots, \beta_{p}$ are regression coefficients to be determined from data.

The ratio $R^{2}$ is used to judge the fit of a linear model to a given body of data [42].

$$
R^{2}=1-\frac{\sum\left(y_{i}-\hat{y}_{i}\right)^{2}}{\sum\left(y_{i}-\bar{y}\right)^{2}}
$$

where $\left(y_{i}-\hat{y}_{i}\right)$ is the residual and $\left(y_{i}-\bar{y}\right)$ is the deviation from the mean.

The ratio $R^{2}$ can be interpreted as the proportion of the total variation in $\mathrm{Y}$ that is accounted for by the variable $\mathrm{X}$. When the model fits the data well, the value of $R^{2}$ will be close to 1 . On the other hand, if the linear model gives a poor fit, the value of $R^{2}$ will be near zero. In this study, IBM SPSS, a statistics software, was used for regression analysis. The statistic results, including $\beta$ and $\mathrm{R}^{2}$ values, were derived from measured data using this tool.

\subsection{Regression Models in Previous Studies}

In previous studies, researchers attempted to find regression models for engine sound design based on variables that can be measured from engine sounds. Researchers at the Honda R\&D Center studied the relationship between Sportiness and variables Level of Order, Tonality-type Metric, and $\triangle \mathrm{RPM}[1]$. They proposed a regression equation for Sportiness.

The semantic differential (SD) test is a widely used method in previous studies for engine sound evaluation. In their study on engine sound analysis, Rhiu et al. [27] developed an SD scale using regression analysis. Krebber et al. conducted SD tests to perform an objective evaluation of a car's interior sound [14].

A two-dimensional positioning method was proposed using the two-dimensional positioning map for engine sound evaluation from previous studies by Bisping [3], [4], Penne [26], Gabriella [10], and Bodden \& Belschner [6]. BMW built its brand identity using a two-dimensional positioning map. Fig. 1 shows an example of a brand sound design for the BMW engine sound [26].

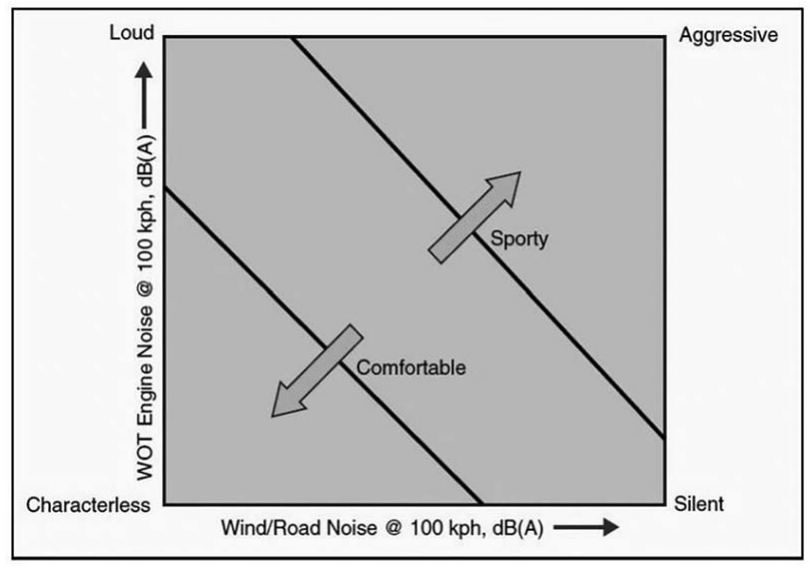

Fig. 1. Example of brand sound design adapted from the study by Penne [26] and Gabriella [10].

Chang et al. [7] studied the relationship between representative adjectives and the metric acoustic parameters of engine sound. They extracted adjectives for sound evaluation from previous studies and derived two representative adjectives that can be used for regression models using factor analysis. Then, they measured the acoustic parameters for 32 engine sound recordings and performed correlation analysis and regression analysis for representative adjectives and acoustic parameters based on a jury test by 42 participants. They proposed a regression model for two representative adjectives, namely Refined and Powerful, based on the analyses results.

Jo et. al. [12] studied a two-dimensional sound quality index for brand sound using neural networks. They extracted two words from previous studies, namely Booming and Rumbling, which are important for brand sound design. Then, they analyzed the relationship between the two words and psychoacoustic parameters including Loudness, Sharpness, Roughness, and Fluctuation using a neural network.

Most previous studies on regression models for engine sound were conducted based on psychoacoustic parameters. Regression models based on psychoacoustic parameters can be used to identify their causal relationships with affects. However, an order-level-based regression model is essential for real ASD development considering that an ASD system is controlled by a tuning tool that adjusts engine order levels.

\section{NEW PARAMETERS FOR REGRESSION MODEL}

\subsection{Frequency Band as a Parameter in Previous Studies}

From prior experiences of sound engineers, causal relationships have been identified between frequency ranges and affects, and sound engineers have applied this knowledge to equalizer settings in the field.

According to studies by Stanley [37], which describe the relationship between semantics and frequency equalization, and studies by Myles [23], which describe the additive and 
subtractive effects of equalization, different sound images from different affects can be derived by adjusting the level of each frequency range. This implies that affects to a sound can be predicted and the sound images can be controlled by intentionally adjusting the levels of the expected frequency ranges.

Some researchers employed frequency bands as parameters for engine sound modeling using low-pass, high-pass, and band-pass filters. In the study by Jo et al. [12], band-pass and low-pass filters were used for Loudness and Roughness measurements in different frequency bands, respectively. They found that the Booming score has a causal relationship with Loudness and Sharpness, which were measured with a low-pass filter, and the Rumbling score has a causal relationship with Loudness, which was measured with a band-pass filter. These parameters were also used in the statistical analysis performed in this study. The results were compared with the analysis results of regression models based on the order-level parameters and are presented in sec. 6 .

\subsection{Possibility of Engine Order as a Parameter}

Nobert et al. compared musical harmony and the structure of engine orders; they subsequently found that the concept of musical intervals can be applied to engine sounds by using engine orders [24]. A musical interval would be perceived from the engine sound by adjusting the level of each engine order.

Yun et al. compared engine sounds and musical harmony by recording the engine sound of a car with a 6-cylinder engine and measuring the frequencies of each engine order at 5,880 RPM [32]. Then, they compared the structure of engine orders to the structure of musical harmony. The results indicate that engine sound has a very similar structure to musical harmony.

\subsection{New Application of Harmonics Pattern to Engine Sound Design}

Dickens et al. [8] noted that the harmonics of a clarinet are composed of odd harmonics, whereas those of a flute are composed of even harmonics. Furthermore, the sound pressure levels of the fundamental frequencies of the clarinet and flute are the highest, and the levels decrease as the number of harmonics increases by odd or even numbers.

Although the level of a frequency range represents partial affects, harmonics patterns generate a general impression of a sound that distinguishes it from others. Different harmonic patterns of each instrument allow people to differentiate the sounds of instruments properly even if the same note is played. The concept of harmonics structure of an instrument can also be applied to engine sound design.

The main order, which has the most energy compared to others, can be considered as the fundamental frequency of engine sound. Subsequently, the harmonics can be derived based on the main order.

There are two benefits of this approach in engine sound analysis. First, reliable parameters, which are used for instrument sound analysis, can be derived. Second, different properties of 4, 6, and 8-cylinder engines can be applied by using a different main order for each case instead of a fixed number of orders.

The harmonics structures of engine sound, which comprise the main order and harmonics of the main order of 4 , 6 , and 8-cylinder engines, are introduced in sec. 2.4. In this study, they are proposed as parameters for the regression model.

\subsection{Main Order and Harmonics as New Parameters}

The result of fast Fourier transform (FFT) analysis shows that the 4, 6, and 8-cylinder engine sounds have different main orders, and the Loudness levels are higher than others. Harmonics in which the order numbers are the results of multiplying the main order by integer numbers were also observed in the FFT analysis results.

Noise, vibration, and harshness (NVH) and acoustical engineers know from experience that the levels of the main order and half orders affect the level of Powerful. Candidates for the parameters were derived based on an interview with NVH experts and acoustical engineers with more than 10 years' experience in the automotive industry. These were the main order and the 2nd, 3rd, and 4th harmonics of the main order. Four half orders near the main order and the 2nd harmonics of the main order were also recommended.

In this study, new parameters, including Sum Main_Main2, H_Main_Main2, and Sum_Main3_Main4, developed based on the Loudness levels of the main order, harmonics of the main order, and half orders, are proposed for a new regression model. Details of each parameter are described in sec. 3.3.2. An additional parameter, Overall Level (SPL without weighing), is introduced below.

\subsection{Overall Level (SPL) as an Additional Parameter}

In addition to the main order and harmonics of the main order, the overall level (SPL), which was considered by Moon et al. [22] as a parameter for engine sound analysis, was used as an additional parameter in this study to build a new regression model.

A-weighted SPL (dBA), in which insensitivity of the ear is applied depending on the frequency range [35], was mainly used in previous studies for engine sound analysis; however, SPL without weighting was used in this study to apply a parameter in which the effects of the bass and treble of engine sound on Refined score can be efficiently reflected simultaneously.

The Loudness curve indicates that the human ear can perceive treble and bass better when the sound level is increased [35]. The results of SPL measurements of engine sounds recorded in this study indicate that the values are in the range of 85-95 $\mathrm{dB}$ (SPL). The improved perception of treble and bass in this range when Loudness is increased can result in different estimations in terms of spectral valance for engine sound.

The benefit of using the overall level as a parameter is that it can simultaneously reflect the causal relationship 
Table 1. List of 27 cars used for jury test.

\begin{tabular}{|c|c|c|c|}
\hline Brand & Model & Group & Cylinder \\
\hline & A-1 & Sporty & 4 \\
\hline \multirow[t]{4}{*}{ A } & A-2 & Luxury & 6 \\
\hline & A-3 & Compact & 4 \\
\hline & B-1 & Sporty & 4 \\
\hline & B-2 & Compact & 4 \\
\hline \multirow[t]{4}{*}{ B } & B-3 & Luxury & 8 \\
\hline & B-4 & Sporty & 8 \\
\hline & B-5 & Sporty & 6 \\
\hline & C-1 & Sporty & 4 \\
\hline \multirow[t]{4}{*}{$\mathrm{C}$} & $\mathrm{C}-2$ & Sporty & 8 \\
\hline & C-3 & Luxury & 8 \\
\hline & D-1 & Luxury & 6 \\
\hline & D-2 & Luxury & 6 \\
\hline \multirow[t]{6}{*}{ D } & D-3 & Luxury & 6 \\
\hline & D-4 & Luxury & 8 \\
\hline & D-5 & Luxury & 6 \\
\hline & D-6 & Compact & 4 \\
\hline & E-1 & Luxury & 6 \\
\hline & E-2 & Compact & 4 \\
\hline \multirow[t]{3}{*}{$\mathrm{E}$} & E-3 & Compact & 4 \\
\hline & E-4 & Compact & 4 \\
\hline & E-5 & Luxury & 6 \\
\hline \multirow[t]{2}{*}{$\mathrm{F}$} & $\mathrm{F}-1$ & Compact & 4 \\
\hline & $\mathrm{F}-2$ & Compact & 4 \\
\hline G & G-1 & Sporty & 8 \\
\hline $\mathrm{H}$ & $\mathrm{H}-1$ & Compact & 4 \\
\hline I & $\mathrm{I}-1$ & Sporty & 8 \\
\hline
\end{tabular}

between two frequency bands (treble and bass) and affective adjectives. The overall level uses Flat weighting (or no weighting). Results of statistical analysis show that overall level has a causal relationship with Refined, and it was used as a parameter for the regression model. Results of statistical analysis of the relationship between Refined and overall level are presented in sec. 5 .

\section{ENGINE SOUND SAMPLE RECORDINGS}

\subsection{Cars for Engine Sound Recordings}

The engine sounds of 27 cars of global car brands (A, B, C, D, E, F, G, H, and I) comprising compact, luxury, and sporty cars were recorded for the jury test. The engine sounds were recorded using a dummy head in the $3 \mathrm{rd}$ gear wide open throttle (WOT) condition. In this study, accelerated engine sounds were used for the jury test to avoid merged dimensions considering the result of previous studies by Kubo et al. [15], [16]. Kubo et al. found that dimensions are merged or cannot be discriminated under a constant speed condition. Table 1 lists the 27 cars used for the jury test.

Information regarding the car models and brand names were not shared with the participants to avoid any preconception of the car brands. Only the names listed in Table 1 were used for the questionnaire during the test.

\subsection{Recording Procedure}

The target car was set on a dynamometer in a semianechoic chamber and a dummy head was installed on the passenger's seat in the test cars, as shown in Fig. 2. A

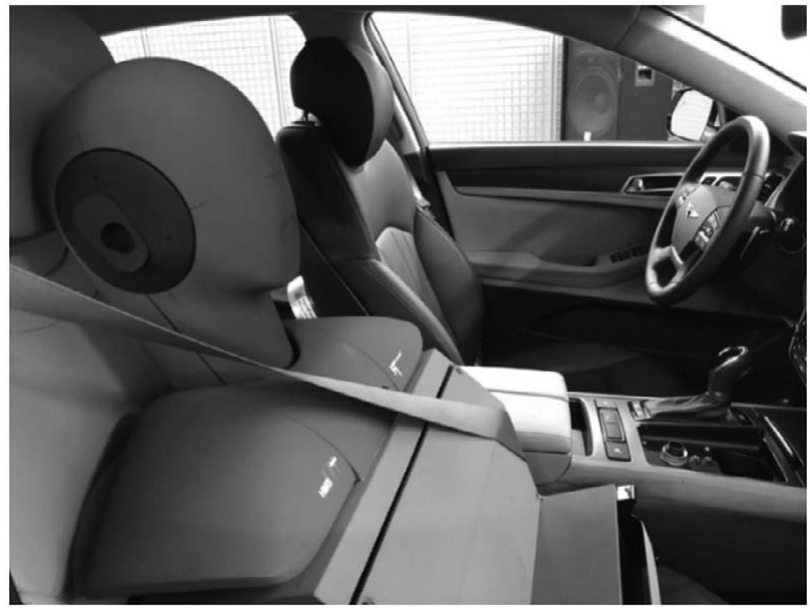

Fig. 2. Example of dummy head setup for engine sound recording.

driver sat on the driver's seat and increased the RPM using the accelerator pedal under the 3rd gear WOT condition.

A Head Acoustics recorder was used to record the engine sounds in the cars while the RPM was increased.

The RPM and engine sound were simultaneously recorded for engine sound analysis. The duration of each engine sound recording was approximately $10 \mathrm{~s}$.

\subsection{Acoustic Parameters}

Two types of acoustic parameters were measured. The first type is based on low-passed/band-passed Loudness, which was used in the study by Jo et al. [12], whereas the second type is based on the order level proposed in this study. The measurement results are analyzed in sec. 6 and compared with those reported by Chang et al. [7], which proposed psychoacoustic parameters for regression model.

\subsubsection{Acoustic Parameters Based on Low-Passed/Band-Passed Loudness Level}

Acoustic parameters based on low-passed/band-passed Loudness levels were used in this study to compare the statistical analysis results with those of the acoustic parameters based on order Loudness level.

Psychoacoustic parameters, including Sharpness (acum), Loudness (sone), Fluctuation strength (max, vacil), and Roughness (max, asper) were measured by referring to the parameters used in the study by Jo et al. [12]. In addition, the overall level (dB SPL) was measured.

Low-pass (LP) and band-pass (BP) filters were used for Loudness measurement, which were also used in a previous study by Jo et al. for Loudness and Roughness measurements [12]. Loudness_LP200 indicates the Loudness level in which a low-pass filter of $200 \mathrm{~Hz}$ was used. Loudness_BP200to500 indicates the Loudness level in which a band-pass filter of $200-500 \mathrm{~Hz}$ was used.

All acoustic parameters were measured in three RPM ranges, namely overall RPM (2,000-5,000 RPM), lower RPM (2,000-3,500 RPM), and higher RPM (3,500-5,000 RPM) ranges for 27 engine sound recordings using the Artemis tool based on the approach proposed by Moon et al. 
Table 2. Acoustic parameters based on low passed/band passed Loudness levels.

\begin{tabular}{ccc}
\hline & Acoustic Parameters & \\
2,000-5,000 RPM & $2,000-3,500$ RPM & $3,500-5,000$ RPM \\
\hline LHrpm_Sharpness & Lrpm_Sharpness & Hrpm_Sharpness \\
LHrpm_Loudness & Lrpm_Loudness & Hrpm_Loudness \\
LHrpm_O.Level & Lrpm_O.Level & Hrpm_O.Level \\
LHrpm_Fluctuation_MAX & Lrpm_Fluctuation_MAX & Hrpm_Fluctuation_MAX \\
LHrpm_Roughness_MAX & Lrpm_Roughness_MAX & Hrpm_Roughness_MAX \\
LHrpm_Loudness_LP200 & Lrpm_Loudness_LP200 & Hrpm_Loudness_LP200 \\
LHrpm_Loudness_BP200to500 & Lrpm_Loudness_BP200to500 & Hrpm_Loudness_BP200to500 \\
\hline
\end{tabular}

[22]. They found that dividing the RPM range by 2 helps to derive the regression model with a higher coefficient of determination $\left(\mathrm{R}^{2}\right)$. All the parameters are listed in Table 2 .

\subsubsection{Acoustic Parameters Based on Order Loudness Level}

The recorded engine sounds for 27 cars were analyzed using the Head Acoustics Artemis tool. The Loudness levels of the main order, 2nd, 3rd, and 4th harmonics for each car engine sound were measured.

For 4-cylinder engine sounds, the Loudness levels of the 2nd, 4th, 6th, and 8th orders, which correspond to the main order, $2 \mathrm{nd}$, 3rd, and 4 th harmonics of the main order, were measured.

For 6-cylinder engine sounds, the Loudness levels of the 3rd, 6th, and 9th orders were measured, whereas the Loudness levels of the 4th, 8 th, 12th, and 16th orders were measured for 8-cylinder engine sounds of the test cars to be used for new acoustic parameters, as listed in Table 3.

The Loudness levels of half orders, which are near the main order and 2nd harmonics, were measured for all the engine sound recordings.

For 4-cylinder engine sounds, the Loudness levels of 1.5th, 2.5th, 3.5th, and 4.5th orders were measured.

For 6-cylinder engine sounds, the Loudness levels of 2.5th, 3.5th, 5.5th, and 6.5th orders were measured. For 8cylinder engine sounds, the Loudness levels of 3.5th, 4.5 th, 7.5 th, and 8.5 th orders were measured.

The order-level measurements were conducted at overall RPM, lower RPM, and higher RPM, similar to measurements conducted for the Acoustic Parameters based on Low-passed/Band-passed Loudness Level. In addition, the overall SPL level was measured in the same RPM ranges.

Table 3 lists the 12 acoustic parameters developed based on order Loudness levels and overall levels from the 27 engine sound recordings.

\section{JURY TEST}

\subsection{Participants}

A total of 36 participants, including 27 males and 9 females, with normal hearing ability and more than five years of driving experience participated in the jury test.

The age of the participants was between 20 and 40 years. People who owned a luxury or sports car were not considered to avoid their preferences.

\subsection{Affective Adjectives and Test Procedure}

The jury test was conducted for 27 engine sound recordings by 36 participants. During the jury test, participants were asked to listen to engine sound recordings and answer a questionnaire. The participants were asked to write the scores for two affective adjectives, namely Powerful and Refined, for each engine sound recording, which were also used in previous studies conducted by Chang et. al. [7] and Moon et al. [22].

The participants listened to 27 engine sound recordings using Sennheiser HD850 headphones and an external sound card in a listening room under a low noise condition (less than $35 \mathrm{dBA}$ ) and then filled the questionnaire. Information regarding the cars, including brand, car type, and number of cylinders, was not shared with the participants during the test to avoid any preconception.

The engine sounds were played in the order of the Latin Square design, and the participants were allowed to repeat the sounds and take a break at any time based on their physical and mental conditions. A 1-min interval was observed between each engine sound, and there was a 10-min break after listening to 14 engine sound recordings. In total, it took approximately $1 \mathrm{~h}$ for each participant to listen to 27 engine sound recordings (including break) and fill the questionnaire

\subsection{Questionnaire}

The questionnaire had two parts. The first part included questions on personal information, such as age, preferred car brand, driving experience, and owned car model. The second part included questions on the scores of Powerful and Refined and overall satisfaction. The participants were asked to fill the questionnaire for 27 cars using the Likert 7-point scale after listening to each engine sound.

\section{RESULTS AND ANALYSIS}

\subsection{Positioning Map for Powerful and Refined}

Fig. 3 shows a two-dimensional positioning map with the Powerful (x) and Refined (y) axes. The map depicts the positions of each engine sound based on the scores of Powerful and Refined from the jury test results.

The two-dimensional positioning map, which was also used by Chang et al. [7] and Jo et al. [12], is a very useful graphic tool to determine the sound characters of current engine sounds and define the future strategy for engine 
Table 3. Acoustic parameters based on sum of order Loudness levels.

\begin{tabular}{|c|c|c|c|c|}
\hline Acoustic Parameters & 4-cylinder & 6-cylinder & 8-cylinder & Unit \\
\hline \multicolumn{5}{|c|}{ Acoustic Parameters for 2,000-5,000 RPM } \\
\hline LHrpm_O.Level & & Overall Level & & $\mathrm{dB}[\mathrm{SPL}]$ \\
\hline LHrpm_Sum_Main_Main2 & $2 \mathrm{nd}+4 \mathrm{th}$ & $3 \mathrm{rd}+6$ th & 4 th +8 th & sone \\
\hline LHrpm_Sum_H.Main_H.Main2 & 1.5 th +2.5 th +3.5 th +4.5 th & 2.5 th +3.5 th +5.5 th +6.5 th & 3.5 th +4.5 th +7.5 th +8.5 th & sone \\
\hline LHrpm_Sum_Main3_Main4 & 6 th +8 th & 9 th +12 th & 12 th +16 th & sone \\
\hline \multicolumn{5}{|c|}{ Acoustic Parameters for 2,000-3,500 RPM } \\
\hline Lrpm_O.Level & & Overall Level & & $\mathrm{dB}[\mathrm{SPL}]$ \\
\hline Lrpm_Sum_Main_Main2 & $2 \mathrm{nd}+4$ th & $3 \mathrm{rd}+6 \mathrm{th}$ & 4 th +8 th & sone \\
\hline Lrpm_Sum_H.Main_H.Main2 & 1.5 th +2.5 th +3.5 th +4.5 th & 2.5 th +3.5 th +5.5 th +6.5 th & 3.5 th +4.5 th +7.5 th +8.5 th & sone \\
\hline Lrpm_Sum_Main3_Main4 & 6 th +8 th & 9 th +12 th & 12 th +16 th & sone \\
\hline \multicolumn{5}{|c|}{ Acoustic Parameters for 3,500-5,000 RPM } \\
\hline Hrpm_O.Level & & Overall Level & & $\mathrm{dB}[\mathrm{SPL}]$ \\
\hline Hrpm_Sum_Main_Main2 & $2 \mathrm{nd}+4 \mathrm{th}$ & $3 \mathrm{rd}+6$ th & 4 th +8 th & sone \\
\hline Hrpm_Sum_H.Main_H.Main2 & 1.5 th +2.5 th +3.5 th +4.5 th & 2.5 th +3.5 th +5.5 th +6.5 th & 3.5 th +4.5 th +7.5 th +8.5 th & sone \\
\hline Hrpm_Sum_Main3_Main4 & 6 th +8 th & 9 th +12 th & 12 th +16 th & sone \\
\hline
\end{tabular}

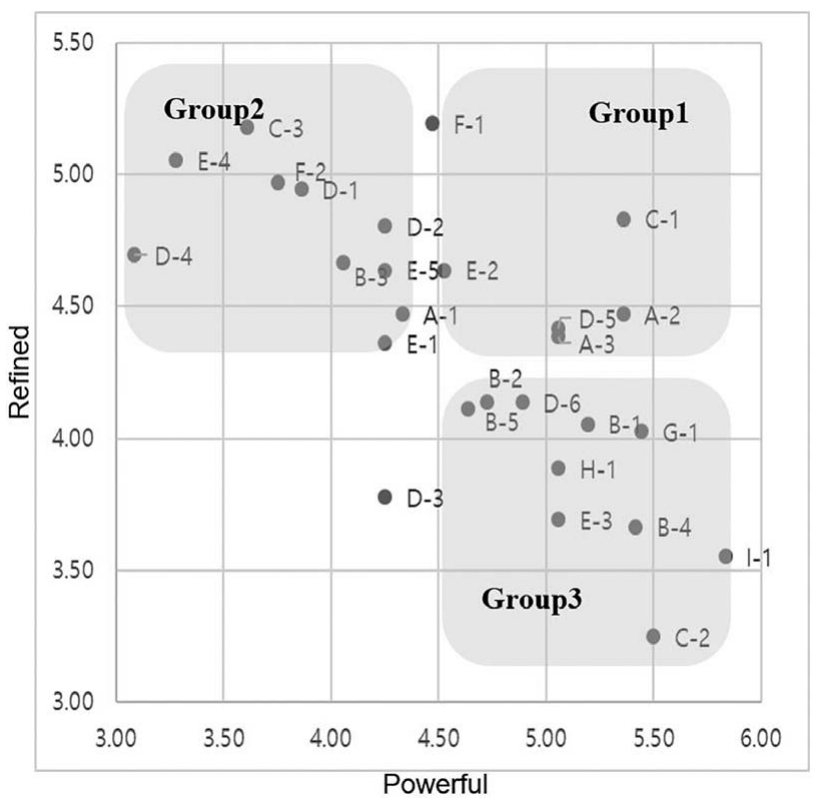

Fig. 3. Two-dimensional positioning map for Powerful (x) and Refined (y) measurement.

sound design. The position of engine sounds on the map can be moved from one location to another by changing the acoustic parameters.

The characters of engine sounds can be grouped using three concepts (Group1, Group2, and Group3) based on the scores of Powerful and Refined as proposed by Moon [21]. For example, engine sounds with high scores for Powerful (higher than 4.5) and Refined (higher than 4.3) can be in Group1, as shown in Fig. 3. Engine sounds with high scores only for Refined (low score for Powerful) or Powerful (low score for Refined) can be in Group2 or Group3.

\subsection{Descriptive Statistics}

\subsubsection{Descriptive Statistics for Parameters Based on Low-Passed/Band-Passed Loudness Level}

Table 4 presents results of the descriptive statistics for the acoustic parameters based on low-passed/band-passed
Loudness obtained from the engine sound recording analysis.

\subsubsection{Descriptive Statistics Parameters Based on Order Loudness Level}

Table 5 presents results of the descriptive statistics for the affective adjectives, which were evaluated in the jury test, and the acoustic parameters based on order of Loudness level.

\subsection{Correlation Analysis}

\subsubsection{Correlation Analysis for Parameters Based on Low-Passed/Band-Passed Loudness Level}

Correlation analysis was performed for 21 acoustic parameters. According to the correlation analysis result for acoustic parameters and affective adjectives, LHrpm _Sharpness $(\mathrm{r}(27)=0.582, p=0.001)$, Hrpm_Loudness $(\mathrm{r}(27)=0.575, p=0.002)$, and Hrpm_Loudness_BP200to $500(\mathrm{r}(27)=0.574, p=0.002)$ demonstrated a strong relationship with Powerful. The numbers in brackets show the results of correlation analysis.

LHrpm_Roughness_MAX (r(27) $=-0.604, p=0.001)$, Lrpm_Roughness_MAX $(\mathrm{r}(27)=-0.596, p=0.001)$, and Hrpm_Roughness_MAX $(\mathrm{r}(27)=-0.599, p=0.001)$ demonstrated a strong negative relationship with Refined. Hrpm_Roughness_MAX exhibited a strong relationship with LHrpm_Roughness_MAX $(\mathrm{r}(27)=1.00, p=0.000)$, and Lrpm_Loudness showed a strong relationship with Lrpm_Loudness_BP200to500 $(\mathrm{r}(27)=0.996, p=0.000)$.

Thus, LHrpm_Roughness_MAX and Lrpm_Loudness BP200to500 were removed from the candidates for the regression model. As a result, 19 acoustic parameters, apart from these two parameters, were used for regression analysis.

\subsubsection{Correlation Analysis for Parameters Based on Order Loudness Level}

Correlation analysis was performed for order level-based acoustic parameters of 27 engine sound recordings to de- 
Table 4. Result of descriptive statistics for parameters based on low passed/band passed Loudness.

\begin{tabular}{|c|c|c|c|c|c|c|}
\hline Name & Min & Max & Mean & Standard Deviation & Skewness & Kurtosis \\
\hline \multicolumn{7}{|c|}{ Acoustic Parameters for 2,000-5,000 RPM (unit) } \\
\hline LHrpm_Sharpness (acum) & 0.85 & 1.57 & 0.99 & 0.14 & 2.816 & 10.186 \\
\hline LHrpm_Loudness (sone) & 13.00 & 62.70 & 22.70 & 10.94 & 2.555 & 7.049 \\
\hline LHrpm_O.Level (dB) & 86.29 & 100.76 & 93.02 & 4.16 & 0.382 & -0.660 \\
\hline LHrpm_Fluctuation_MAX (vacil) & 0.1 & 0.23 & 0.03 & 0.05 & 3.185 & 10.611 \\
\hline LHrpm_Roughness_MAX (asper) & 1.88 & 4.18 & 2.37 & 0.45 & 2.709 & 9.358 \\
\hline LHrpm_Loudness_LP200 (sone) & 7.08 & 39.00 & 13.49 & 7.12 & 2.390 & 6.167 \\
\hline LHrpm_Loudness_BP200to500 (sone) & 7.48 & 38.50 & 13.62 & 7.38 & 2.461 & 6.035 \\
\hline \multicolumn{7}{|c|}{ Acoustic Parameters for 2,000-3,500 RPM (unit) } \\
\hline Lrpm_Sharpness (acum) & 0.74 & 1.34 & 0.88 & 0.11 & 2.601 & 9.127 \\
\hline Lrpm_Loudness (sone) & 10.50 & 58.40 & 19.00 & 10.44 & 2.641 & 7.801 \\
\hline Lrpm_O.Level (dB) & 83.30 & 99.64 & 90.51 & 4.51 & 0.464 & -0.389 \\
\hline Lrpm_Fluctuation_MAX (vacil) & 0.05 & 0.83 & 0.19 & 0.18 & 2.343 & 6.068 \\
\hline Lrpm_Roughness_MAX (asper) & 1.24 & 3.67 & 1.72 & 0.50 & 2.523 & 8.005 \\
\hline Lrpm_Loudness_LP200 (sone) & 6.01 & 41.00 & 11.93 & 7.64 & 2.581 & 7.629 \\
\hline Lrpm_Loudness_BP200to500 (sone) & 5.93 & 36.00 & 11.32 & 6.91 & 2.571 & 6.881 \\
\hline \multicolumn{7}{|c|}{ Acoustic Parameters for 3,500-5,000 RPM (unit) } \\
\hline Hrpm_Sharpness (acum) & 0.91 & 1.81 & 1.08 & 0.17 & 3.041 & 11.608 \\
\hline Hrpm_Loudness (sone) & 15.50 & 66.9 & 26.49 & 11.59 & 2.413 & 6.010 \\
\hline Hrpm_O.Level (dB) & 88.05 & 102.55 & 94.46 & 4.15 & 0.301 & -0.760 \\
\hline Hrpm_Fluctuation_MAX (vacil) & 0.04 & 0.39 & 0.13 & 0.09 & 1.548 & 1.530 \\
\hline Hrpm_Roughness_MAX (asper) & 1.88 & 4.18 & 2.37 & 0.46 & 2.702 & 9.302 \\
\hline Hrpm_Loudness_LP200 (sone) & 8.15 & 36.80 & 15.05 & 6.76 & 2.095 & 4.492 \\
\hline Hrpm_Loudness_BP200to500 (sone) & 9.04 & 40.80 & 15.94 & 7.93 & 2.333 & 5.186 \\
\hline
\end{tabular}

termine the correlation between 12 acoustic parameters and two affective adjectives, namely Refined and Powerful.

LHrpm_Sum_H.Main_H.Main2 $(\mathrm{r}(27)=0.559, p=$ 0.002), Hrpm_Sum_Main_Main2 $(\mathrm{r}(27)=0.536, p=$ 0.004), and Hrpm_Sum_H.Main_H.Main2 $(\mathrm{r}(27)=0.585, p$ $=0.001$ ) showed a strong relationship with Powerful. However, only Hrpm_Sum_H.Main_H.Main2 $(\mathrm{r}(27)=-0.532$, $p=0.004)$ showed a strong relationship with Refined.

In terms of correlation between acoustic parameters, LHrpm_Sum_H.Main_H.Main2 showed a strong relationship with Lrpm_Sum_H.Main_H.Main2 $(\mathrm{r}(27)=0.987$, $p=0.000)$ and Hrpm_Sum_H.Main_H.Main2 $(\mathrm{r}(27)=$ $0.988, p=0.000$ ).
Therefore, LHrpm_Sum_H.Main_H.Main2 was removed as a candidate for the regression model; only 11 acoustic parameters were considered for regression analysis. Results of regression analysis and regression model, which are based on 11 acoustic parameters, are presented in Sec. 5.4.3 and 5.4.4.

\subsection{Regression Analysis and Regression Model 5.4.1 Regression Models}

Regression analysis was performed for Refined and Powerful to determine their relationship with two types of parameters, namely low-passed/band-passed Loudness-based

Table 5. Result of descriptive statistics for affective adjectives and parameters based on order Loudness.

\begin{tabular}{|c|c|c|c|c|c|c|}
\hline Name & Min & Max & Mean & Standard Deviation & Skewness & Kurtosis \\
\hline \multicolumn{7}{|l|}{ Affective Adjectives } \\
\hline Powerful & 3.08 & 5.83 & 4.61 & 0.72 & -0.391 & -0.549 \\
\hline Refined & 3.25 & 5.19 & 4.37 & 0.52 & -0.284 & -0.683 \\
\hline \multicolumn{7}{|c|}{ Acoustic Parameters for 2,000-5,000 RPM (unit) } \\
\hline LHrpm_O.Level (dB) & 86.29 & 100.76 & 93.02 & 4.16 & 0.382 & -0.669 \\
\hline LHrpm_Sum_Main_Main2 (sone) & 7.21 & 41.77 & 15.30 & 8.30 & 1.635 & 2.915 \\
\hline LHrpm_Sum_H.Main_H.Main2 (sone) & 9.64 & 53.13 & 20.82 & 10.98 & 1.815 & 3.218 \\
\hline LHrpm_Sum_Main3_Main4 (sone) & 1.98 & 15.44 & 5.81 & 3.06 & 1.367 & 2.534 \\
\hline \multicolumn{7}{|c|}{ Acoustic Parameters for 2,000-3,500 RPM (unit) } \\
\hline Lrpm_O.Level $(\mathrm{dB})$ & 83.30 & 99.64 & 90.51 & 4.51 & 0.464 & -0.389 \\
\hline Lrpm_Sum_Main_Main2 (sone) & 5.88 & 39.40 & 14.20 & 8.43 & 1.556 & 1.943 \\
\hline Lrpm_Sum_H.Main_H.Main2 (sone) & 8.24 & 49.26 & 18.06 & 10.70 & 1.883 & 3.155 \\
\hline Lrpm_Sum_Main3_Main4 (sone) & 1.85 & 14.96 & 5.51 & 3.11 & 1.267 & 2.002 \\
\hline \multicolumn{7}{|c|}{ Acoustic Parameters for 3,500-5,000 RPM (unit) } \\
\hline Hrpm.O.Level $(\mathrm{dB})$ & 88.05 & 102.55 & 94.46 & 4.15 & 0.301 & -0.760 \\
\hline Hrpm_Sum_Main_Main2 (sone) & 7.89 & 36.00 & 16.03 & 7.84 & 1.108 & 0.570 \\
\hline Hrpm_Sum_H.Main_H.Main2 (sone) & 11.04 & 59.70 & 23.73 & 11.51 & 1.707 & 3.310 \\
\hline Hrpm_Sum_Main3_Main4 (sone) & 2.09 & 15.82 & 6.25 & 3.34 & 1.225 & 1.515 \\
\hline
\end{tabular}


parameters and order-level-based parameters, to develop regression models.

Two regression models were derived from the regression analysis for each parameter. Multicollinearity was not observed as the values of the variance influence factor (VIF) were less than 10 for all regression models.

\subsubsection{Regression Analysis for Parameters Based on Low-Passed/Band-Passed Loudness Level}

Nineteen parameters, apart from LHrpm Roughness_MAX and Lrpm_Loudness_BP200to500, which were removed from the correlation analysis, were considered for regression analysis.

A stepwise method was employed as the statistical analysis tool. Powerful is described by Hrpm_O.Level, Hrpm_Loudness_LP200, and Hrpm_Fluctuation_MAX. The coefficient of determination $\left(\mathrm{R}^{2}\right)$ for Powerful is 0.797 (Adjusted $\mathrm{R}^{2}=0.770, \mathrm{~F}=9.219, p=0.006$ ). Refined is described by LHrpm_O.Level and Hrpm_Loudness BP200to500. The coefficient of determination $\left(\mathrm{R}^{2}\right)$ for Refined is 0.700 (Adjusted $\mathrm{R}^{2}=0.675, \mathrm{~F}=0.719, p=0.405$ ), as presented in Table 6 .

\subsubsection{Regression Analysis for Parameters Based on Order Loudness Level}

Eleven acoustic parameters, apart from LHrpm _Sum_H.Main_H.Main2, which was removed from correlation analysis, were used for regression analysis. A stepwise method was employed as the statistical analysis tool.

Table 7 summarizes the result of the regression analysis for parameters based on order level. Powerful is described by Hrpm_Sum_H.Main_H.Main2, Hrpm_O. Level, and Hrpm_Sum_Main_Main2. The coefficient of determination $\left(\mathrm{R}^{2}\right)$ for Powerful is 0.807 (Adjusted $\mathrm{R}^{2}=$ $0.782, \mathrm{~F}=13.608, p=0.001)$. Refined is described by Hrpm_Sum_H.Main_H.Main2, LHrpm.O.Level, and Lrpm_Sum_Main3_Main4. The coefficient of determination $\left(\mathrm{R}^{2}\right.$ ) for Refined is 0.721 (Adjusted $\mathrm{R}^{2}=0.684, \mathrm{~F}=7.543$, $p=0.011)$.

\subsubsection{Explanation for Regression Model Based on Order Loudness Level}

Table 7 presents the regression model, which reflects the causal relationship between Powerful/Refined and parameters based on order Loudness level.

The regression model shows that increasing the levels of the sum of half orders in the higher RPM range (3,5005,000 RPM) near the main order increases the score of Powerful, whereas decreasing the sum of half orders increases the score of Refined, as expected based on interview with $\mathrm{NVH}$ experts presented in Sec. 2.4. NVH and acoustical engineers know from experience that the levels of the main order and half orders affect the level of Powerful.

Fig. 4 shows examples of two engine sounds (4-cylinder engine from two different brands, A-1 and C-1) with the score of Powerful, which illustrates the contribution of Hrpm_Sum_Main_Main2 to Powerful in the regression model presented in Table 7.

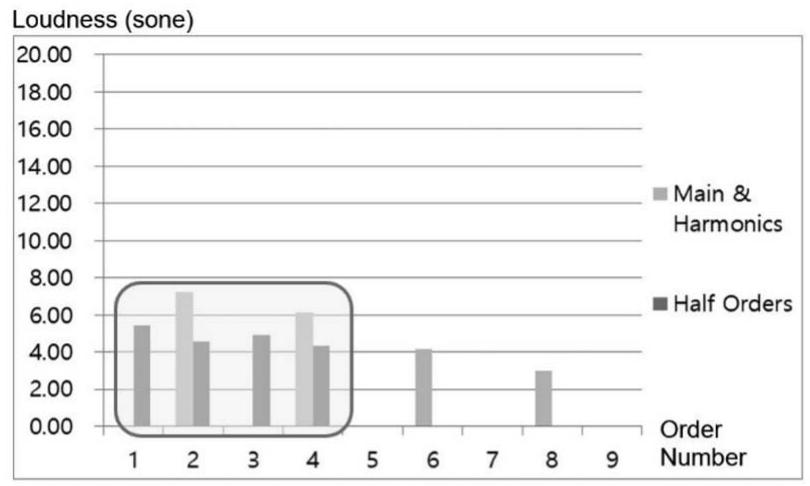

(a) Case 1 (A-1): Powerful $=4.33$

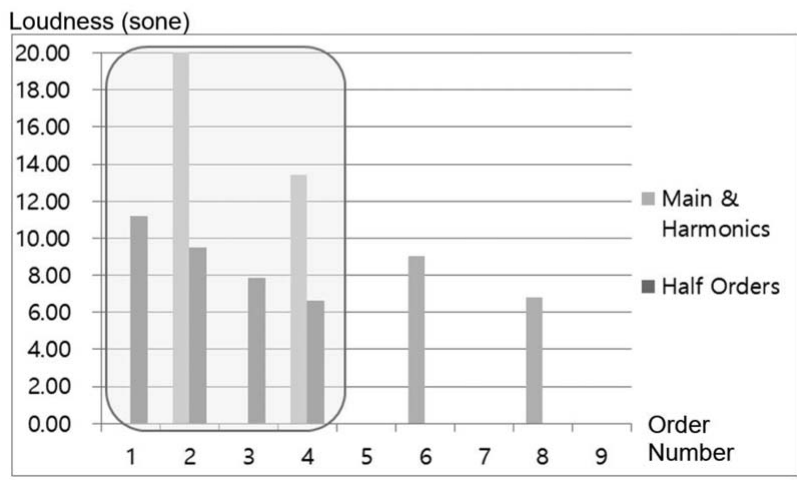

(b) Case 2 (C-1): Powerful $=5.36$

Fig. 4. Example of harmonics and half orders in the higher RPM range $(3,500-5,000$ RPM).

In the regression model presented in Table 7(a), the sum of the main order and $2 \mathrm{nd}$ harmonics of the main order in the higher RPM range (3,500-5,000 RPM) has a positive causal relationship with Powerful. This indicates that increasing the levels of the main order or 2 nd harmonic of the main order increases the score of Powerful. Fig. 4 shows that the main order level and the 2nd harmonics of the main order from $\mathrm{C}-1$ are higher than those from A-1. This indicates that the results can predict Powerful based on the regression model presented in Table 7(a).

Fig. 5 shows examples of two engine sounds (4-cylinder engine from two different brands, A-1 and C-1) with the score of Refined, which illustrates the contribution of Lrpm_Sum_Main3_Main4 to Refined in the regression model presented in Table 7 .

The spectral balance of the engine sound can be adjusted by controlling the levels of lower- and higher-order groups, which changes the Refined score of the engine sound. The main order and 2nd harmonics of the main order are considered as a lower-order group based on interview with NVH and acoustical experts. In addition, the 3rd and 4th orders are considered as a higher-order group. Orders in which the number is higher than four are not considered as candidates for analysis in this study. During the interview for this study, NVH experts agreed that the 16th order, 4th har- 
Table 6. Result of analysis for parameters based on low-passed/band-passed Loudness level.

(a) Powerful

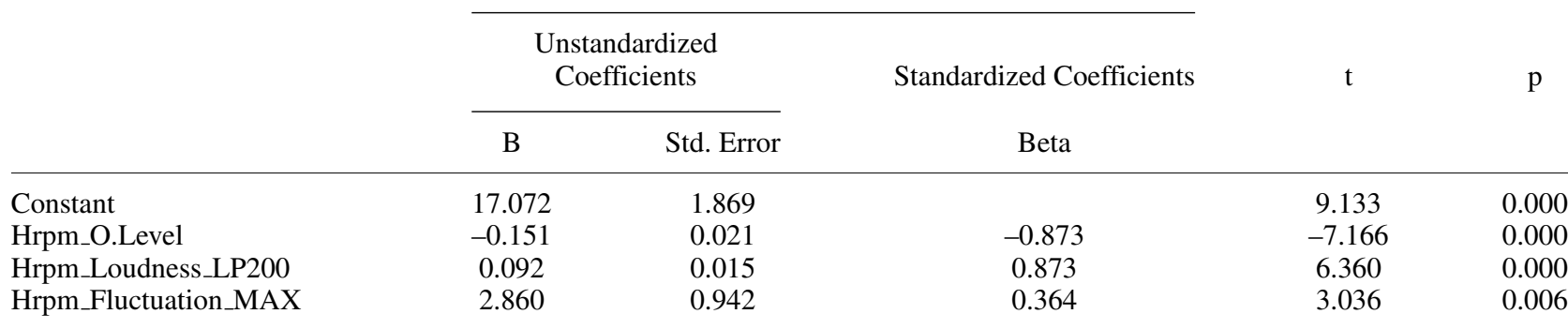

(b) Refined

\begin{tabular}{lcccc}
\cline { 2 - 5 } & \multicolumn{2}{c}{$\begin{array}{c}\text { Unstandardized } \\
\text { Coefficients }\end{array}$} & Standardized Coefficients & $\mathrm{t}$ \\
\cline { 2 - 5 } & $\mathrm{B}$ & Std. Error & Beta & -2.522 \\
\hline Constant & -4.055 & 1.608 & & 0.019 \\
LHrpm_O.Level & 0.103 & 0.018 & 0.819 & 0.000 \\
Hrpm_Loudness_BP200to500 & -0.71 & 0.010 & -1.079 & -7.400 \\
\hline
\end{tabular}

monics for an 8-cylinder is a very high number for engine sound analysis.

In the regression model presented in Table 7(b), the sum of the 3rd and 4th harmonics in the lower RPM range (2,000-3,500 RPM) has a positive causal relationship with Refined; that is, increasing the levels of the 3rd or 4th harmonics of the main order in the higher rpm range increases the score of Refined.

Fig. 5 shows that the 3rd and 4th harmonics of the main order from $\mathrm{C}-1$ are higher than those from A-1. The result can be predicted based on the regression model presented in Table 7(b). According to the measurement result of the 3rd and 4th harmonics of the main order, the Refined score of $\mathrm{C}-1$ is expected to be higher than that of A-1.
The overall level is another important parameter in the regression model in this study. In the regression model presented in Table 7, the overall level (SPL) has a negative relationship with Powerful in the higher RPM range $(3,500-$ 5,000 RPM) and a Positive causal relationship with Refined in the overall RPM range (2,000-5,000 RPM). When the overall SPL level is increased, the Loudness curve is expected to change.

Increasing the overall level in the range of 85-95 $\mathrm{dB}$ (SPL) increases the score of Refined in the overall RPM range. The Loudness curve explains the human perception for frequency depending on the SPL. High and low frequencies in a higher SPL are more perceived than those of lower SPL.

Table 7. Result of regression analysis for parameters based on order Loudness level.

\begin{tabular}{|c|c|c|c|c|c|}
\hline & \multicolumn{3}{|c|}{ (a) Powerful } & \multirow{3}{*}{$\mathrm{t}$} & \multirow{3}{*}{$\mathrm{p}$} \\
\hline & \multicolumn{2}{|c|}{$\begin{array}{l}\text { Unstandardized } \\
\text { Coefficients }\end{array}$} & \multirow{2}{*}{$\begin{array}{c}\text { Standardized Coefficients } \\
\text { Beta }\end{array}$} & & \\
\hline & $\mathrm{B}$ & Std. Error & & & \\
\hline Constant & 17.352 & 1.853 & & 9.363 & 0.000 \\
\hline Hrpm_Sum_H.Main_H.Main2 & 0.033 & .010 & 0.527 & 3.138 & 0.005 \\
\hline Hrpm.O.Level & -0.153 & .021 & -0.890 & -7.384 & 0.000 \\
\hline Hrpm_Sum_Main_Main2 & 0.061 & .017 & 0.671 & 3.689 & 0.001 \\
\hline
\end{tabular}

(b) Refined

\begin{tabular}{lcccr}
\cline { 2 - 4 } & \multicolumn{2}{c}{$\begin{array}{c}\text { Unstandardized } \\
\text { Coefficients }\end{array}$} & Standardized Coefficients & $\mathrm{t}$ \\
\cline { 2 - 3 } & $\mathrm{B}$ & Std. Error & Beta & -1.264 \\
\hline Constant & -2.054 & 1.625 & & 0.219 \\
Hrpm_Sum_H.Main_H.Main2 & -0.059 & 0.008 & -1.301 & 0.000 \\
LHrpm_O.Level & 0.079 & 0.019 & 0.632 & 4.306 \\
Lrpm_Sum_Main3_Main4 & 0.082 & 0.030 & 0.491 & 0.000 \\
\hline
\end{tabular}


Table 8. Comparison of three regression models.

Psychoacoustic (Chang

et al. [7])

Powerful
LP/BP Loudness

Powerful Refined
Order Level

Powerful Refined

Regression Model

\begin{tabular}{|c|c|c|c|c|c|c|}
\hline Method of Analysis & Stepwise & & Stepwise & & Stepwise & \\
\hline $\mathrm{R}^{2}$ score & 0.659 & 0.643 & 0.797 & 0.700 & 0.807 & 0.721 \\
\hline F-value & 50.800 & 8.170 & 9.219 & 0.719 & 13.608 & 7.543 \\
\hline p-value & 0.000 & 0.000 & 0.006 & 0.405 & 0.001 & 0.011 \\
\hline
\end{tabular}

Parameters

Number of Parameters

Number of Parameters based on Order

Number of Parameters based on SPL

Number of Psychoacoustic Parameters

Significant Level

Dependency on Number of Cylinders

$\begin{array}{lll}2 & 7 & 3 \\ 0 & 3 & 0 \\ 0 & 1 & 1 \\ 2 & 3 & 2 \\ <0.05 & <0.05 & <0.05 \\ \text { No } & \text { No } & \text { No }\end{array}$

No

2
0
1
1
$<0.05$
No

\begin{tabular}{ll}
3 & 3 \\
2 & 2 \\
1 & 1 \\
0 & 0 \\
$<0.05$ & $<0.05$ \\
Yes & Yes \\
\hline
\end{tabular}

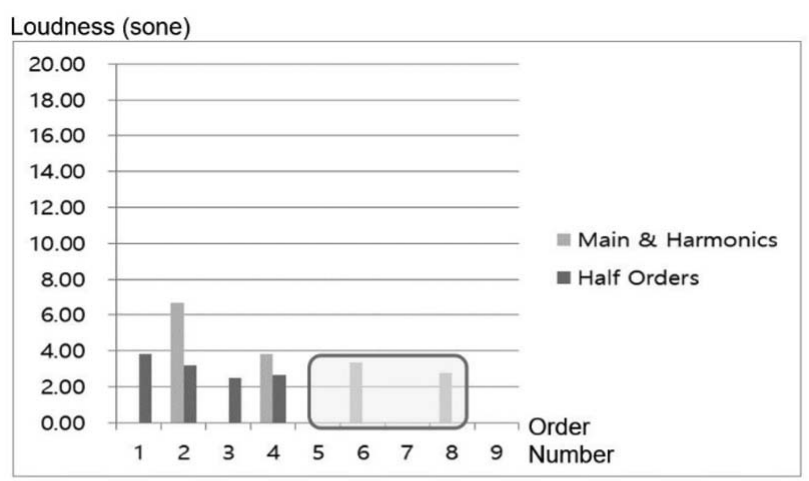

(a) Case 1 (A-1): Refined $=4.47$

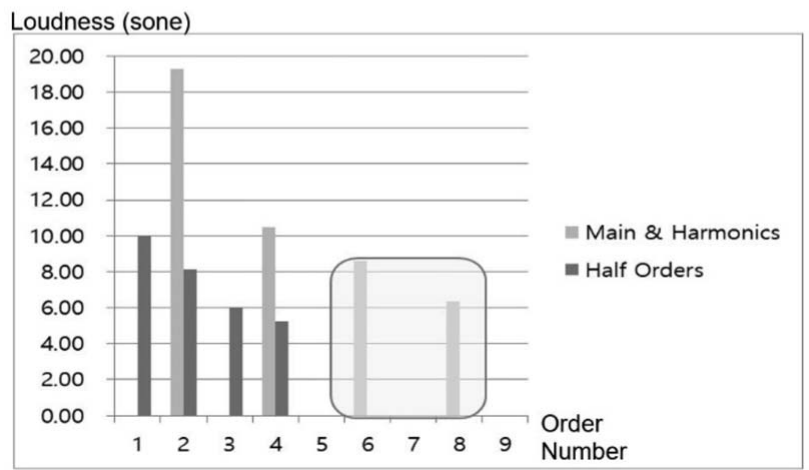

(b) Case 2 (C-1): Refined 4.83

Fig. 5. Example of harmonics and half orders in the lower RPM range (2,000-3,500 RPM).

\section{CONCLUSION}

This study proposed an engine sound model for engine sound design based on the main order of engine sound and its harmonics. First, a jury test was conducted for 27 engine sound recordings by 36 participants to judge Powerful and Refined scores.
Second, two types of new acoustic parameters were proposed, namely low-passed (LP)/band-passed (BP) Loudness level-based parameters, which were based on a previous study by Jo et al. [12], and order Loudness levelbased parameters, which were formulated in this study. The acoustic parameters were measured from engine sound recordings using an engine order measurement tool, and the overall levels (SPL) were also measured for each engine sound. All the parameters were measured in three RPM ranges, namely overall RPM (2,000-5,000 RPM), lower RPM (2,000-3,500 RPM), and higher RPM (3,500-5,000 RPM).

Third, statistical analysis was performed for two affective adjectives and two types of new acoustic parameters. The causal relationships between the affective adjectives (Powerful and Refined) and two types of new acoustic parameters were analyzed by descriptive analysis, correlation analysis, and regression analysis. In the end, two regression models were derived for Powerful and Refined using two types of parameters.

Two regression models were derived for each parameter from the regression analysis. Multicollinearity was not observed as the values of VIF were less than 10 for all regression models. All regression equations were statistically significant at the 0.05 level, indicating that the two models are statistically meaningful.

Details of the regression model proposed by Chang et al. [7] are presented in Table 8 (Psychoacoustic) for comparison with the two regression models proposed in this study. The regression model based on order Loudness level has the highest $\mathrm{R}^{2}$ score ( 0.807 for Powerful, 0.721 for Refined) of the three models in the table. This indicates that the regression model based on order Loudness level best reflects the behavior of the variables.

The regression models in Table 8 have $2-3$ parameters, except the regression model for Refined proposed by Chang et al. [7]. The regression model proposed by Chang et al. has seven parameters for Refined, which is very high compared 
with the other models. Too many parameters can make it difficult to understand the causal relationship between independent and dependent variables. NVH and acoustic engineers recommend 2-3 parameters for a regression model to apply the parameters to a real ASD system.

Regression models based on order Loudness level comprise two parameters based on order Loudness level and one parameter based on SPL, whereas other regression models comprise mainly psychoacoustic parameters.

Psychoacoustic parameters have been used in previous studies for ASD development; however, an order levelbased parameter is needed for a real ASD system controlled by engine order levels. Therefore, the result of the regression model based on order Loudness level can be easily applied to an ASD system considering that most ASD systems are controlled by changing the order Loudness levels of engine sound.

In general, the regression models based on order Loudness level yielded the best results in Table 7. They had the highest $\mathrm{R}^{2}$ scores compared with the other models and comprise order-based parameters that can easily be applied to ASD systems. Further development using the regression model proposed in this study will reduce time and effort.

The findings of this study also align with the insight of NVH engineers introduced in sec. 2.4. They have been implementing causal relationship between Powerful/Refined and order Loudness level based on their experiences.

The jury test in this study was conducted for only Korean participants. The results obtained in this study can be used for other regions; however, some existing studies showed different preferences for engine sound, depending on the nationality or background of the participants.

To develop a general regression model that can be used for all regions, a global jury test would be required. Dedicated regression models for each region can be considered if the results for each region vary to a considerable degree.

\section{REFERENCES}

[1] Y. Ishii, N. W. Alt, and S. Jochum, "Sound Design under the Aspects of Musical Harmonic Theory," presented at the SAE 2003 Noise \& Vibration Conference and Exhibition (2003), https://doi.org/10.4271/2003-01-1508.

[2] R. Bisping, "Emotional Effect of Car Interior Sounds: Pleasantness and Power and Their Relation to Acoustic Key Features," presented at the 1995 Noise and Vibration Conference-P-291 (1995), https://doi.org/ 10.4271/951284.

[3] R. Bisping, "Car Interior Sound Quality: Experimental Analysis by Synthesis," Acta Acust. united with Acust., vol. 83, pp. 813-818 (1997 Sep.). https://doi.org/ $10.4271 / 971976$

[4] R. Bisping, S. Giehl, and M. Vogt, "A Standardized Scale for the Assessment of Car Interior Sound Quality," presented at the 1997 Noise and Vibration Conference-P3091997 Noise and Vibration Conference-P-309 (1997), https://doi.org/10.4271/971976.

[5] M. Blommer, S. Amman, and N. Otto, "The Effect of Powertrain Sound on Perceived Vehicle Performance," presented at the 1997 Noise and Vibration Conference-P3091997 Noise and Vibration Conference-P-309 (1997), https://doi.org/10.4271/971983.

[6] M. Bodden and T. Belschner, "Comprehensive Active Sound Design Part 1: Electric and Combustion Vehicle," presented at the Inter-Noise Inter-Noise 2014 (2014).

[7] K. Chang, S. Kim, D. Park, and S. Moon, "A Research on Brand Sound Positioning and Implementing with Active Sound Design," presented at the Noise and Vibration Conference and Exhibition Noise and Vibration Conference and Exhibition (2017), https:// doi.org/10.4271/2017-01-1754.

[8] P. Dickens, R. France, J. Smith, and J. Wolfe, "Clarinet Acoustics: Introducing a Compendium of Impedance and Sound Spectra," Acoust. Aust., vol. 35, no. 1, pp. 1-19 (2007 Apr.).

[9] S. J. Elliot and P. A. Nelson, "The Minimum Active Control of Sound," Electron. Commun. Eng. J., vol. 2, no. 4, pp. 127-136 (1990 Aug.). https://doi.org/ 10.1049/ecej: 19900032

[10] C. Gabriella, "Automotive Sound Quality - Powertrain, Road and Wind Noise," Sound Vib., vol. 43, no. 4, pp. 16-24 (2009 Apr.).

[11] M. Iwai, K. Ito, and T. Yamashita, "A Support System for Development of Sporty Sound and Its Application," presented at the SAE 2003 Noise \& Vibration Conference and Exhibition SAE 2003 Noise \& Vibration Conference and Exhibition (2003), https://doi.org/10.4271/2003-01-1400.

[12] B. O. Jo, S. K. Lee, D. C. Park, M. S. Lee, and S. G. Jung, "New Development of Two-Dimensional Sound Quality Index for Brand Sound in Passenger Cars," Korean Soc. Noise Vib. Eng., vol. 16, no. 5, pp. 457-469 (2006 May). https://doi.org/10.5050/ksnvn.2006.16.5.457

[13] J. Kim, S. Ryu, J. Jang, M. Lee, S. Kim, D. Park, and Y. Lee, "Implementation of Active Sound Design System for Improvement of Engine Sound Quality," Korean Soc. Noise Vib. Eng., vol. 2015, pp. 280-281 (2015 Apr.).

[14] W. Krebber, M. Adams, F. Brandl, N. Chouard, K. Genuit, T. Hempel, R. Hofe, G. Irato, P. Ponseele, B. SaintLoubry, B. Schulte-Fortkamp, R. Sttek, and R. Weber, "Objective Evaluation of Interior Car Sound - the OBELICS Project," DAGA 2000, Fortschritte der Akustik, 186 (Oldenburg, Germany, 2000).

[15] N. Kubo, V. Mellert, R. Weber, and J. Meschke, "Categorization of Engine Sound," presented at the 33rd International Congress and Exposition on Noise Control Engineering 33rd International Congress and Exposition on Noise Control Engineering (2004).

[16] N. Kubo, V. Mellert, R. Weber, and J. Meschke, "Engine Sound Perception - Apart from So-called Engine Order Analysis," presented at the CFA/DAGA 2004 CFA/DAGA 2004 (2004).

[17] S. Kuwano and S. Namba, "Dimensions of Sound Quality and Their Measurement," presented at the 17th International Congress on Acoustics 17th International Congress on Acoustics (2001).

[18] S. Lee, B. Kim, and D. Park, "Objective Evaluation of the Rumbling Sound in Passenger Cars Based on 
an Artificial Neural Network," In Proceedings of the Institution of Mechanical Engineers, Part D, J. Automob. Eng., vol. 219, no. 4, pp. 457-469 (2005 Apr.). https://doi.org/ $10.1243 / 095440705 \times 11112$

[19] Y. Lee, J. Kim, S. Ryu, S. Kim, and D. Park, "Implementation of Active Sound Enrichment Control for Improving Engine Sound Quality Inside the Cabin of a Passenger Car," Korean Soc. Noise Vib. Eng., vol. 26, no. 2, pp. 195-202 (2016 Apr.). https://doi.org/10.5050/ksnve. 2016.26.2.195

[20] A. Melka and P. Pelant, "Psychoacoustic Methods Applied to SKODA Passenger Car Development Programmes: Intentions and First Experiences," Acta Acust. united with Acust., vol. 85, no. 5 pp. 657-660 (1999 Sep.).

[21] S. Y. Moon, "Development of a Sonic Branding Process for Designing Automobile Engine Sound Based on Affective Engineering Approach," Diploma thesis, Seoul National University (2019).

[22] S. Y. Moon, D. H. Park, D. G. Park, W. J. Kim, M. H. Yun, and D. C. Park, "A Study on Affective Dimensions to Engine Acceleration Sound Quality Using Acoustic Parameters," Appl. Sci., vol. 9, no. 3, pp. 604 (2019 Jan.). https://doi.org/10.3390/app9030604

[23] B. Myles, "Space is the Place, Part II," Electronic Musician, pp. 26 (2009 Jan.).

[24] W. Nobert, N. Alt, and S. Jochum, "Sound Design under the Aspects of Musical Harmonic Theory," presented at the SAE 2003 Noise \& Vibration Conference and Exhibition SAE 2003 Noise \& Vibration Conference and Exhibition (2003), https://doi.org/10.4271/2003-01-1508.

[25] D. Park, E. Jo, S. Hong, and M. Csakan, "Development of Personalized Engine Sound System Using Active Sound Design Technology," presented at the SAE 2015 Noise and Vibration Conference and Exhibition SAE 2015 Noise and Vibration Conference and Exhibition (2015), https://doi.org/10.4271/2015-01-2216.

[26] F. Penne, "Shaping the Sound of the Next Generation BMW," presented at the International Conference of Noise and Vibration Engineering ISMA 2004 International Conference of Noise and Vibration Engineering ISMA 2004 (2004).

[27] I. Rhiu, S. Kwon, M. Yun, and D. Park M, "Analysis of Relationship between Personality and Customer Satisfaction on a Vehicle Exhaust Sound," Int. J. Ind. Eng., vol. 23, no.1, pp. 68-82 (2016 Jul.).

[28] R. Schirmacher, "Active Design of Automotive Engine Sound," presented at the 112th Convention of the Audio Engineering Society 112th Convention of the Audio Engineering Society (2002 Apr.), convention paper 5544.
[29] A. Sköld, D. Västfjäll, and M. Kleiner, "Perceived Sound Character and Objective Properties of Powertrain Noise in Car Compartments," Acta Acust. united with Acust., vol. 91, no. 2, pp. 349-355 (2005 Mar.).

[30] D. Vastfjall, M. A. Gulbol, M. Kleiner, and T. Garling, "Affective Evaluations of and Reactions to Exterior and Interior Vehicle Auditory Quality," J. Sound Vib., vol. 255, no. 3, pp. 501-518 (2002). https://doi.org/ 10.1006/jsvi.2001.4166

[31] J. Yoshida and T. Igata, "Influence of Impression of Vehicle Styling on Loudness of Acceleration Sounds in Cabin," J. Acoust. Soc. Am., vol. 130, no. 1, pp 19-14 (2011 June). https://doi.org/10.1121/1.3599004

[32] T. Yun, D. Park, G. Won, and T. Im, "Musical Harmonic Theory Analysis of Automotive Interior Noise," presented at the 2012 Spring Conference, The Korean Society for Noise and Vibration Engineering 2012 Spring Conference, The Korean Society for Noise and Vibration Engineering (2012).

[33] A. Zeiter and P. Zeller, "Psychoacoustical Modeling of Sound Attributes," presented at the SAE 2006 World Congress \& Exhibition SAE 2006 World Congress \& Exhibition (2006), https://doi.org/10.4271/2006-01-0098.

[34] S. M. Kuo and D. R. Morgan, Active Noise Control Systems (John Wiley \& Sons, New York, New York, 1996).

[35] T. D. Rossing, R. F. Moore, and P. A. Wheeler, The Science of Sound, 3rd ed. (Pearson Press, London, United Kingdom, 2014).

[36] J. F. Moore, An Introduction to the Psychology of Hearing, 6th ed. (Brill Press, London, United Kingdom, 2013).

[37] R. A. Stanley, Audio in Media, 9th ed. (Wadsworth Press, Belmont, Californiam 2011).

[38] E. Zwicker and H. Fastle, Psychoacoustics - Facts and Models, 2nd ed. (Springer-Verlag, Berlin, Germany, 1999). https://doi.org/10.1007/978-3-662-09562-1

[39] Bimmerpost, ASD of BMW F10 M5, http://www. bimmerpost.com.

[40] Standard Weighting Networks, https://www.ocn. org.au/guide/244-weighting-dba-and-c-weighting-dbc.

[41] FFT Analysis of Sound of Violin, Trumpet and Guitar, http://www.biostat.jhsph.edu/ ririzarr.

[42] S. Chattejee, and A. S. Hadi, Regression Analysis by Example (Wiley, New York, New York, 2012).

[43] D. C. Montgomery, E. A. Peck, and G. G Vining, Introduction to Linear Regression Analysis (Wiley, New York, New York, 2011). 


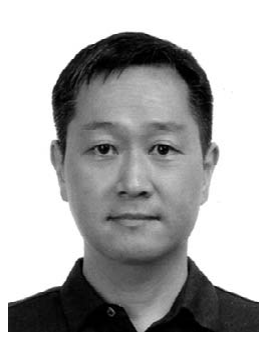

Soyoun Moon

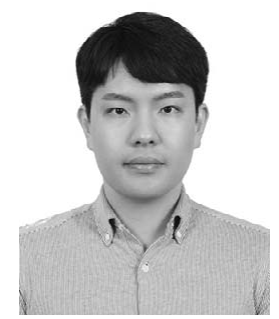

Sunghwan Park

\section{THE AUTHORS}

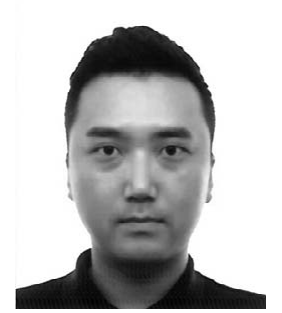

Donggun Park

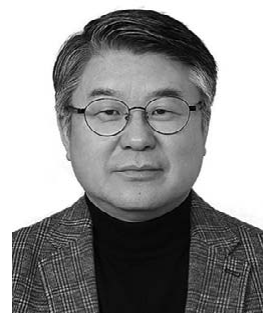

Myung Hwan Yun

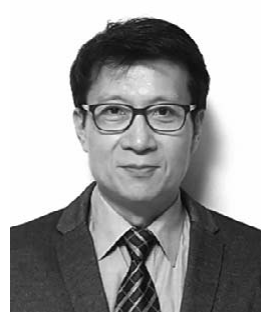

Kyoung-Jin Chang

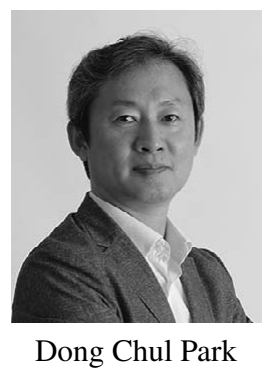

Soyoun Moon studied Electronics Engineering at Ajou University and Music Composition at Seoul National University, Korea. He received a master's degree in Business in 2011 and a PhD in Industrial Engineering in 2018 at Seoul National University. His research interests include an auditory user interface (AUI) and active sound design (ASD). He participated in several car OEM research projects, including AUI and ASD development in a car. In 2002, he founded SoundSketch Co. in Seoul, a music production company, and worked as a recording engineer. SoundSketch, Inc. was incorporated to expand the business to consulting for sound system design and room acoustics in 2005 . He then worked as a senior sound consultant for the company. In 2012, he joined ARKAMYS, a French software company, which provides sound enhancement solutions to automotive industry. He worked as a senior audio expert and performed audio sound tunings for mass-production cars. He became a country manager of ARKAMYS KOREA in 2018 to lead a technical team of the Korean branch office. In 2020, he joined Harman as a manager, leading the Acoustic Sound Engineering team in Korea. He is a member of the AES Technical Committee for Automotive Audio.

Sunghwan Park received a BS degree in electrical engineering from POSTECH, Pohang, Korea, and a BA degree in psychology from Yonsei University, Seoul, Korea, and MS and PhD degrees in Cognitive Science from Seoul National University, Seoul, Korea. He is a researcher at Samsung Electronics. His research interests include usercentered design process and cognitive and social factors in product design and planning.

Donggun Park received a BS degree in industrial engineering from Purdue University, West Lafayette, Indiana, USA, in 2012. He is a PhD candidate in the Department of Industrial Engineering at Seoul National University. His research interests include user experience and human factors in product design.

Myung Hwan Yun received BS and MS degrees in in- dustrial engineering from Seoul National University, Seoul, Korea, and $\mathrm{ahD}$ in industrial and manufacturing engineering from Penn State University, University Park, Pennsylvania, USA. He is a Professor at the Department of Industrial Engineering and Institute of Industrial Systems Innovation, Seoul National University. His research interests include human factors in product design and affective product design.

Kyoung-Jin Chang studied mechanical engineering at Yonsei University, Korea. He received a $\mathrm{PhD}$ in Automotive Vibration Analysis and Structural Dynamic Modification in 1999. Since then, he has been working for Hyundai Motor Company as an expert on Vibration, Noise, and Sound Quality. Currently, he is a Global R\&D Master at Hyundai Motor Group. His goal is to develop the new technologies of improving automotive NVH and sound quality. His research areas are sound design, active control, and passive control. In the sound design field, he has designed the target sound level of each component, such as intake, exhaust, engine, etc., by considering brand sound identity and using the transfer path analysis and statistical methods. In the active control field, he has developed new active noise cancellation and generation system of engine sound using electrodynamic actuators and so forth. In the passive control field, he has been developing a new body panel based on acoustic metamaterial to improve $\mathrm{NVH}$ issues.

Dong Chul Park studied mechanical engineering at Seoul National University, Korea. He received $\mathrm{PhD}$ degrees in Noise \& Vibration in 1996. He has been working for Hyundai Motor Company as NVH and Sound Quality specialist since 1996. Currently, he is a research fellow at Hyundai Motor Group and director of the Sound Design Research Lab. His major working field is product sound designing for the engine, electric warning sound, and operational sound. He also takes charge of active sound designing and an active sound control system for combustion and electric vehicles for the mass product solution. His research areas are psychoacoustics, new sound designing methods, and making a strategy for brand sound. 